\title{
MicroRNA Expression Profiling of Lung Cancer with Differential Expression of the RON Receptor Tyrosine Kinase
}

\author{
Huilin Ou $\left(\mathbb{D},{ }^{1}\right.$ Keda Chen $\mathbb{D D}^{2}$ and Hongcheng Wu $\mathbb{D D}^{1}$ \\ ${ }^{1}$ Ningbo Medical Centre, Li Huili Hospital Affiliated of Ningbo University, Ningbo 315040, China \\ ${ }^{2}$ Shulan International Medical College, Zhejiang Shuren University, Hangzhou 310015, China
}

Correspondence should be addressed to Hongcheng Wu; doctorwu1967@126.com

Received 16 June 2021; Accepted 8 September 2021; Published 24 September 2021

Academic Editor: Yuan Seng Wu

Copyright (c) 2021 Huilin Ou et al. This is an open access article distributed under the Creative Commons Attribution License, which permits unrestricted use, distribution, and reproduction in any medium, provided the original work is properly cited.

Background. The Ron receptor tyrosine kinase (RON) can act as a protooncogene and may play a prominent role in the initiation and development of lung cancer. microRNAs (miRNA) are master regulators of gene expression through direct or indirect regulation, and impact all aspects of cell biology. Methods. Nonsmall-cell lung cancer (NSCLC) samples and small-cell lung cancer (SCLC) were stratified based on RON expression to identify miRNA profiles associated with RON expression levels, differentially expressed miRNA regulated by RON were screened out, and their biological behavior was analyzed. Results. miRNA expression was most significantly affected by cancer type, and we found 85 miRNAs that were significantly differentially expressed between NSCLC and SCLC. There were 46 miRNAs differentially expressed between high RON expressing NSCLC compared to low RON expressing NSCLC. Biological processes and pathways found to be significantly influenced by RON expression included epithelialmesenchymal transition (EMT) and activation of the PI3K-Akt and MAPK signaling pathways. Conclusions. These data may provide the basis for a novel strategy to characterize lung cancer by RON expression and microRNA genotyping.

\section{Introduction}

Lung cancer is the leading cause of cancer-related deaths in the world, and mutations and deregulation of many proteins and pathways, including EGFR, ALK, ROS1, and MET, play a significant role in lung cancer occurrence and development $[1,2]$. Better identification and utilization of these disease-related biomarkers could lead to improvement in precision treatment of lung cancer, ultimately reducing disease incidence, lowering disease recurrence, and improving survival and the quality of life for lung cancer patients.

RON is located at the chromosome 3 p21.3 region, a region that has tumor suppressor activity and undergoes frequent loss of heterozygosity in human lung and breast cancers. The mature RON is a $180 \mathrm{kDa}$ heterodimer composed of a $40 \mathrm{kDa}$ extracellular chain and a $150 \mathrm{kDa}$ transmembrane $\beta$-chain. Stimulation of RON by its ligand, MSP, promotes invasive growth of epithelial cells, resulting from the integration of a number of input pathways, including epithelial-mesenchymal transition (EMT), cellcell dissociation ("scattering"), and extracellular matrix growth and invasion $[3,4]$.

RON is primarily expressed in cells of epithelial origin including lung cells. Several lung cancer cell lines overexpresses Ron and variant isomers, and normal lung tissue exhibits minimal expression of RON compared to adjacent tumor tissue. These data suggest that RON expression may be related to the occurrence and development of both NSCLC and SCLC and could be used as a prognostic indicator for lung cancer patients [5]. Regulation of how RON expression and splicing is deregulated in NSCLC and SCLC requires further characterization. The frequency and intensity of RON and phospho-RON expression in NSCLC is lower than in SCLC, and the $\beta$-RON isoform (around $150 \mathrm{kDa}$ ) was found to be the dominant isoform in NSCLC, compared to the $120 \mathrm{kDa}$ isoform in SCLC. Furthermore, northern blot analysis verified that the RON gene is normally transcribed in the lungs. These data suggest that characterizing mechanisms regulating RON gene expression are of 
great significance to improve understanding of lung tumor occurrence and development [6].

microRNAs (miRNAs) are small noncoding RNAs, $\sim 18-25$ nucleotides in length, that regulate gene expression at the posttranscriptional level by hybridizing to target mRNAs, and either inhibiting gene translation or promoting degradation of messenger RNAs (mRNA) by miRNAs are thought to regulate at least $30 \%$ of human gene expression and are involved in essential biological processes, including cell-cycle control, cell lineage fate decision, cell survival, tissue patterning, vascular development, immune control, and metabolism [7]. miRNAs act as oncogenes or tumor suppressors in various tumors, including lung cancer [8].

RON and miRNAs are closely related to the occurrence and development of lung cancer; however, it is unclear how miRNAs interact with RON to facilitate lung cancer progression. In vitro, RON mutations that alter miRNA expression induce oncogenic and metastatic potential. miRNA profiles are significantly affected by RON expression in pancreatic cancer, indicating the potential role of miRNAs in tumor invasion and metastasis [9]. In the present study, we compare miRNA profiles of lung cancer samples with differential expression of RON in order to investigate how RON regulates miRNA expression in both NSCLC and SCLC.

\section{Materials and Methods}

2.1. MicroRNA Expression Analysis. This research has been carried out in accordance with the World Medical Association Declaration of Helsinki and was approved by the Institutional Review Board (CWO) of Medical School of Ningbo University, Ningbo, China (2020-YXY-0035). All subjects provided written informed consent. Nine NSCLC samples and nine SCLC samples were analyzed by miRNA microarray. Each group was classified based on RON expression levels, no expression, low expression, and high expression, with three samples representing each level. miRNA expression patterns were compared between NSCLC and SCLC samples with different RON expression levels. The expression of RON was determined by Western blot and immunohistochemistry.

2.2. Immunohistochemistry of Lung Tissue. Fragments of lung tissue were incubated with anti-RON monoclonal antibodies $\mathrm{Zt} / f 2$ overnight at $4^{\circ} \mathrm{C}$, followed by the DAKO Envision DAB System visualization reagents (DAKO, Denmark). All slides were counterstained with hematoxylin. RON expression was assessed using a semiquantitative scoring system.

2.3. miRNA Expression by Microarray. Total RNA was harvested using TRIzol (Invitrogen) and the miRNeasy mini kit (QIAGEN), according to manufacturer's instructions. RNA quantity was determined using a NanoDrop 1000 instrument. RNA was labeled using the miRCURY ${ }^{\mathrm{TM}} \mathrm{Hy}^{{ }^{\mathrm{TM}}}$ / $\mathrm{Hy}^{\mathrm{TM}}$ Power labeling kit and hybridized onto the miRCURY ${ }^{\mathrm{TM}}$ LNA Array (v.16.0). The slides were washed and scanned using an Axon GenePix 4000B microarray scanner.

Scanned images were imported into GenePix Pro 6.0 software (axon) for grid alignment and data extraction. miRNAs with intensity $\geq 50$ in all samples were selected for calculating a normalization factor after averaging the replicated miRNAs. After normalization using median normalization, volcano plot filtering was used to identify significantly differentially expressed miRNAs. Hierarchical clustering was performed to visualize distinct miRNA expression profiling among samples.

2.4. miRNA-mRNA Coexpression Network Analysis. Network diagram analysis of miRNAs and mRNAs revealed regulatory relationships between miRNAs and their target genes. Target prediction information was derived from the following three resources: miRWalk (http://mirwalk.umm. uni-heidelberg.de/), miRDB (http://mirdb.org/), and TargetScan (http://www.targetscan.org/vert_60/). Predicted targets were filtered by identifying targets overlapping from the different prediction resources. miRNAs and predicted target genes were analyzed using Cytoscape software, and pathway enrichment was analyzed using the DAVID web resource (https://david.ncifcrf.gov/).

\section{Statistical Methods}

One-way analysis of variance was applied to assess differential expression of miRNAs between groups, and Benjamini-Hochberg FDR correction was applied, in addition to Tukey's honestly significant difference (HSD) post hoc test. A $p$ value of $<0.05$ was considered to indicate a statistically significant difference.

\section{Results}

miRNA expression profiling was performed for NSCLC and SCLC samples with different RON expression levels. miRNA expression levels were more greatly influenced by tumor type than by RON expression levels.

Comparison of miRNA expression between NSCLC and SCLC identified 85 significantly differentially expressed miRNA genes. Among these, 55 (65\%) were overexpressed in SCLC vs. NSCLC, and 30 (35\%) were underexpressed. miRNAs with fold-change with $\geq 1.5$-fold overexpression or $\leq 0.5$-fold underexpression are given in Table 1 and Figure 1 . In addition, RON overexpression was associated with discrete miRNA expression patterns (Table 2), particularly in NSCLC patients (Table 3), compared to RON nonexpressing samples; hierarchical clustering was performed to show the distinct miRNA expression profiling among samples (Figure 2).

In order to discern the major miRNA genes associated with RON overexpression in NSCLC, differentially expressed miRNA genes were obtained after comparing the RON high-expressing and RON nonexpressing NSCLC samples, and a gene coexpression network was constructed. Using the miRWalk, miRDB, and TargetScan miRNA target prediction databases, 1827 overlapping target genes were 
TABLE 1: Differentially expressed miRNAs with $\geq 1.5$-fold overexpression or $\leq 0.5$-fold underexpression in SCLC vs. NSCLC samples.

\begin{tabular}{lccccc}
\hline ID & Name & Fold-change $\geq 1.5$ SCLC vs. NSCLC & ID & Name & Fold-change 1.5 SCLC vs. NSCLC \\
\hline 11040 & hsa-miR-29b-3p & 8.051603 & 11260 & hsa-miR-151a-5p & 0.41632 \\
28191 & hsa-miR-30e-5p & 5.304497 & 46228 & hsa-miR-320c & 0.59677 \\
11000 & hsa-miR-200a-3p & 4.301609 & 42630 & hsa-miR-140-3p & 0.26368 \\
10946 & hsa-miR-141-3p & 3.426177 & 19582 & hsa-miR-106b-5p & 0.50352 \\
10923 & hsa-miR-107 & 3.120694 & 148668 & hsa-miR-378a-3p & 0.28814 \\
10967 & hsa-miR-16-5p & 3.002912 & & & \\
148038 & hsa-miR-3679-3p & 2.81699 & & \\
145996 & hsa-miR-205-3p & 2.803615 & & \\
31026 & hsa-miR-101-3p & 2.425494 & & \\
10138 & hsa-miR-130a-3p & 2.058103 & & \\
10977 & hsa-miR-183-5p & 2.040765 & & \\
145676 & hsa-miR-30e-3p & 1.961315 & & \\
42739 & hsa-miR-339-5p & 1.858763 & & \\
27217 & hsa-miR-34a-5p & 1.760212 & & \\
\hline
\end{tabular}

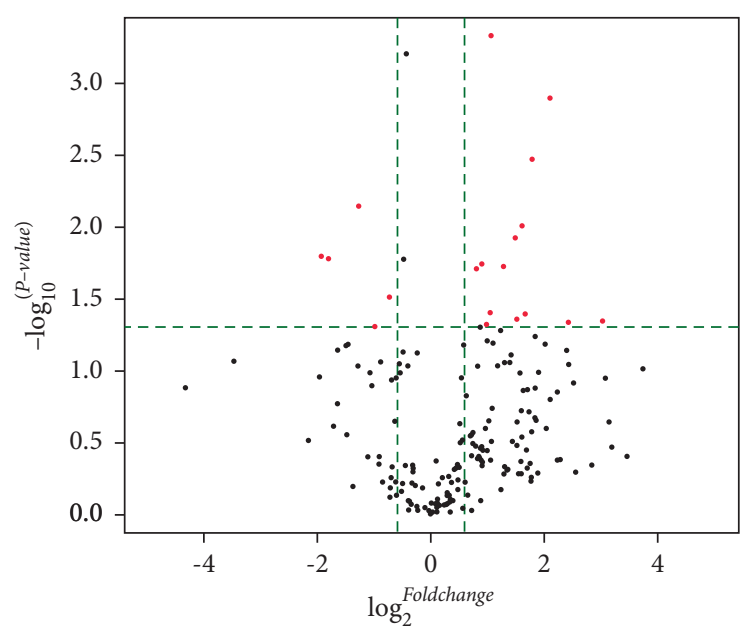

FIGURE 1: Differential expression of miRNAs in NSCLC and SCLC. Volcano plot of differentially expressed miRNAs between SCLC and NSCLC samples. The vertical lines correspond to 1.5 -fold overexpression and underexpression, respectively. The horizontal line represents a $p$ value of 0.05 . The red points in the plot represents differentially expressed miRNAs which met the threshold for statistical significance.

TABLE 2: miRNAs with statistically significant differential expression $\geq 1.5$-fold overexpression or $\leq 0.5$-fold underexpression in high RON expressing lung cancer samples compared to RON nonexpressing samples.

\begin{tabular}{|c|c|c|c|c|c|}
\hline ID & Name & $\begin{array}{c}\text { Fold-change } \geq 1.5 \text { overexpression in } \\
\text { RON high-expressing vs. RON } \\
\text { nonexpressing }\end{array}$ & ID & Name & $\begin{array}{c}\text { Fold-change } \leq 0.5 \text { underexpression in } \\
\text { RON high-expressing vs. RON } \\
\text { nonexpressing }\end{array}$ \\
\hline 145859 & hsa-miR-33a-5p & 2.36 & 28302 & $\begin{array}{c}\text { hsa-miR-27b- } \\
3 p\end{array}$ & 0.49 \\
\hline 148642 & hsa-miR-1246 & 1.59 & 13138 & Hy3 & 0.39 \\
\hline 11000 & $\begin{array}{c}\text { hsa-miR-200a- } \\
3 p\end{array}$ & 1.55 & & & \\
\hline 10936 & $\begin{array}{c}\text { hsa-miR-130b- } \\
3 p\end{array}$ & 1.55 & & & \\
\hline 11260 & $\begin{array}{c}\text { hsa-miR-15la- } \\
5 \mathrm{p}\end{array}$ & 1.55 & & & \\
\hline
\end{tabular}

identified for the 10 most significantly differentially expressed miRNAs. A regulatory network between the overlapping target genes and their upstream miRNAs was constructed using Cytoscape (Figure 3). In order to identify the potential mechanism by which RON may promote
NSCLC through miRNA-mediated regulation, the 1827 predicted target genes were then subjected to DAVID pathway enrichment analysis. This pathway enrichment revealed that RON-related miRNAs are predicted to target genes that significantly affect pathways involved in 
TABLE 3: Differentially expressed miRNAs with $>1.5$-fold overexpression or 0.5 -fold underexpression in high RON expressing NSCLC samples compared to RON nonexpressing NSCLC samples.

\begin{tabular}{|c|c|c|c|c|c|}
\hline ID & Name & $\begin{array}{c}\text { Fold-change } \geq 1.5 \text { RON high-expressing } \\
\text { vs. RON nonexpressing NSCLC }\end{array}$ & ID & Name & $\begin{array}{c}\text { Fold-change } \leq 0.5 \text { RON high-expressing } \\
\text { vs. RON nonexpressing NSCLC }\end{array}$ \\
\hline 46921 & hsa-miR-1290 & 7.57 & 13138 & hsa-let-7c-3p & 0.24 \\
\hline 148642 & hsa-miR-1246 & 7.28 & 147162 & $\begin{array}{c}\text { hsa-miR-31- } \\
5 p\end{array}$ & 0.33 \\
\hline 25611 & spike_control_v2_19 & 4.1 & 147512 & hsa-let-7a & 0.34 \\
\hline 11052 & hsa-miR-21-5p & 3.29 & 147506 & Hy3 & 0.42 \\
\hline 27318 & spike_control_v2_23 & 3.2 & 28302 & $\begin{array}{l}\text { hsa-miR- } \\
320 \mathrm{e}\end{array}$ & 0.46 \\
\hline 145859 & hsa-miR-33a-5p & 2.41 & 11040 & $\begin{array}{l}\text { hsa-miR- } \\
27 b-3 p\end{array}$ & 0.46 \\
\hline 145950 & hsa-miR-33b-5p & 2.3 & 147691 & $\begin{array}{l}\text { hsa-miR- } \\
29 b-3 p\end{array}$ & 0.47 \\
\hline 11260 & hsa-miR-151a-5p & 1.99 & 11030 & $\begin{array}{l}\text { hsa-miRPlus- } \\
\text { A1015 }\end{array}$ & 0.47 \\
\hline 10936 & hsa-miR-130b-3p & 1.89 & 46801 & $\begin{array}{c}\text { hsa-miR- } \\
106 a-5 p\end{array}$ & 0.49 \\
\hline 11000 & hsa-miR-200a-3p & 1.52 & 17503 & $\begin{array}{c}\text { hsa-miR-590- } \\
5 \mathrm{p}\end{array}$ & 0.49 \\
\hline
\end{tabular}

tumorigenesis. The top 4 significantly enriched signaling pathways were pathways in cancer, the PI3K-Akt signaling pathway, the MAPK signaling pathway, endocytosis, and proteoglycans in cancer (Figure 4). In addition, the top 10 overexpressed and top 10 underexpressed miRNAs and their major targets which might have key function were predicted from published data (Figure 5). Overexpression or underexpression of miRNAs detected by the microarray chip is represented by an upward (red) or a downward (blue) arrow, respectively. Changes of target genes expression levels, which were inversely correlated with the expression of the miRNAs that target them, are also represented by a down (blue) or up (red) arrow.

\section{Discussion}

Lung cancer is one of the most common cancers and a leading cause of cancer-related deaths. The identification of critical genetic factors leading to cellular transformation, including EGFR, ALK, and Ros1, has resulted in significant improvements in therapy for advanced lung cancer based on radiotherapy and chemotherapy and has improved the prognosis and quality of life for patients with lung cancer $[1,2]$.

The RON receptor tyrosine kinase is an oncogene that plays an important role in the development of lung cancer. RON overexpression can induce a complex genetic program that results in cell dissociation, migration, and extracellular matrix invasion, which may be important in several tumor types, including lung cancer. The extent of RON overexpression varies widely among different lung cancer cells and between different subtypes of lung cancers. The frequency and intensity of RON expression in different lung cancer subtypes is not well characterized, and the specific mechanism by which RON overexpression contributes to the pathogenesis of different lung cancer subtypes remains unknown. R. Kanteti et al. reported that RON expression in
NSCLC was lower than in primary and secondary SCLC tumors [6]. Reports from M. Wang confirm that RON overexpression is common in lung adenocarcinoma, suggesting that RON may initiate oncogenic programs and plays an important role in the pathogenesis of lung adenocarcinoma $[10,11]$.

miRNAs play important roles in lung tumor progression. Using high-throughput RNA sequencing and bioinformatics, many tumor-related miRNAs and their predicted targets that are oncogenes or tumor suppressor genes have been reported. In this study, miRNA expression patterns in RON high-expressing and RON nonexpressing lung cancer samples were profiled, and we predicted potential gene-miRNA interactions with a systematic bioinformatics approach. Our study demonstrates that the type of tumors, such as SCLC or NSCLC, has the greatest impact on differential miRNA expression patterns. Within subgroups, RON expression had a greater impact on overexpression or underexpression of miRNAs in NSLCL samples than in SLCL samples.

46 differentially expressed miRNA genes in RON highexpressing NSCLC vs. RON nonexpressing NSCLC samples were identified; most of these miRNAs have reported functions in cancer. In addition, we also identified some putative genetic interactions involving networks of miRNAs, such as miR-33a-5p, hsa-miR-33b-5p, hsa-miR-31-5p, and miR-106a-5p. For example, miR-106a-5p has been reported to inhibit the migration and invasion of renal cell carcinoma through targeting PAK5. It has also been reported that miR16a-5p acts as an onco-miRNA by targeting PTEN, E2F3, and E2F5. The Akt/mTOR and PI3K/AKT signaling pathways were also directly regulated by MiR-106a-5p [12, 13].

The most significantly overexpressed miRNA between RON high-expressing and RON nonexpressing NSCLC samples was hsa-miR-1290. hsa-miR-1290 directly targets a number of genes, including NAT1, INPP4B, SOCS4, IRF2, and hMSH2, and activates the JAK/STAT3 and PI3K/AKT 


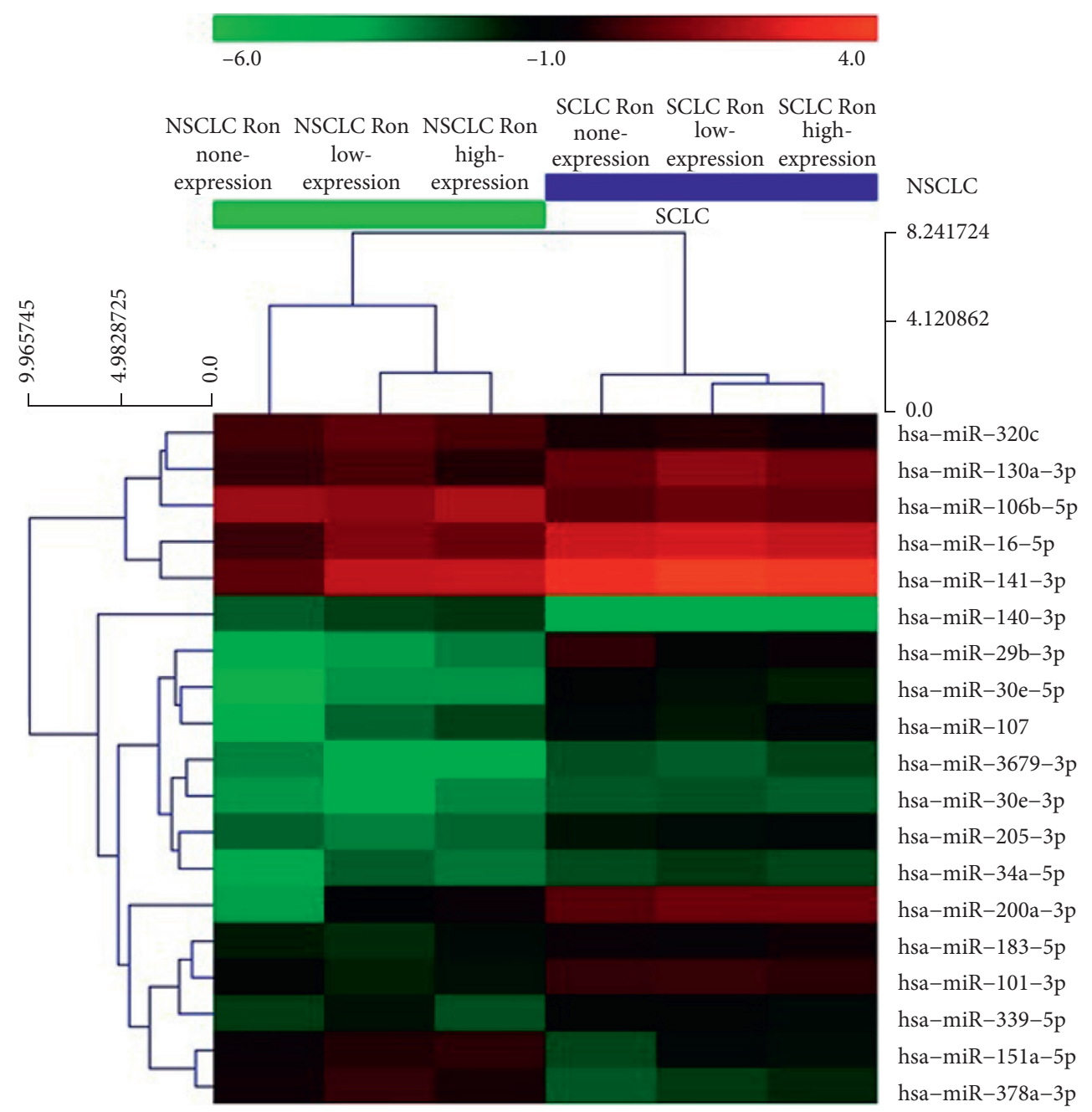

FIGURE 2: Heat map of miRNA expression in SCLC and NSCLC samples. The heat map diagram depicts the results of the two-way hierarchical clustering of miRNAs and sample type. Each row represents a miRNA and each column represents different sample type. The miRNA clustering tree is shown on the left, and the sample clustering tree appears at the top. The color scale shown at the top illustrates the relative expression level of a miRNA in the certain slide: red color represents a high relative expression level; green color represents a low relative expression level.

signaling pathways. Inhibition of miR-1290 resulted in a decrease in stemness markers and EMT markers in NSCLC. Anti-miR-1290 treatment suppressed proliferation, sphereformation, colony formation, and invasion of NSCLC cells in vitro $[14,15]$.

The overexpressed miRNA, hsa-miR-1246, is closely related to the occurrence and development of p53 family tumors. Inhibition of miR-1246 in NSCLC resulted in decreased stemness markers and EMT markers. Anti-miR1246 treatment suppressed the proliferation, sphere-formation, colony formation, and invasion of NSCLC. Furthermore, genes repressed by miR-1246 include PRL36A, GLIPR1, HAS2, NCKAP5, MT1G, CYP4F11, CCNG2, and THBS2 [14, 16, 17].

miR-21-5p promotes peritoneal metastasis through EMT in gastric cancer, increases the proliferation, migration, and invasion of colon cancer by downregulating Tiam1, promotes cell migration and invasion in esophageal cancer by targeting PDCD4, induces metastasis of human cervical carcinoma cells, and can be used as a biomarker to predict the recurrence of digestive system tumors $[18,19]$.

hsa-miR-151a is overexpressed in primary NSCLC and induces proliferation, migration, and EMT as an oncomiRNA by targeting E-cadherin mRNA. hsa-miR-151a also promotes metastasis and functions synergistically with FAK to inhibit RhoGDIA. In addition, miR-151a-5p targets SOCS5 and activates downstream JAK2/STAT3 signaling $[20,21]$.

hsa-miR-130b, an onco-miRNA, is directly regulated by NF- $\kappa \mathrm{B}$ and sustains NF- $\kappa \mathrm{B}$ activation by decreasing cylindromatosis expression. hsa-miR-130b-3p also downregulates PTEN expression, which promotes the proliferation, migration, invasion, and cytoskeletal rearrangement through the activation of PI3K and integrin $\beta 1$ signaling pathways. Moreover, miR-130b-3p inhibitors induced apoptosis [22-24]. 


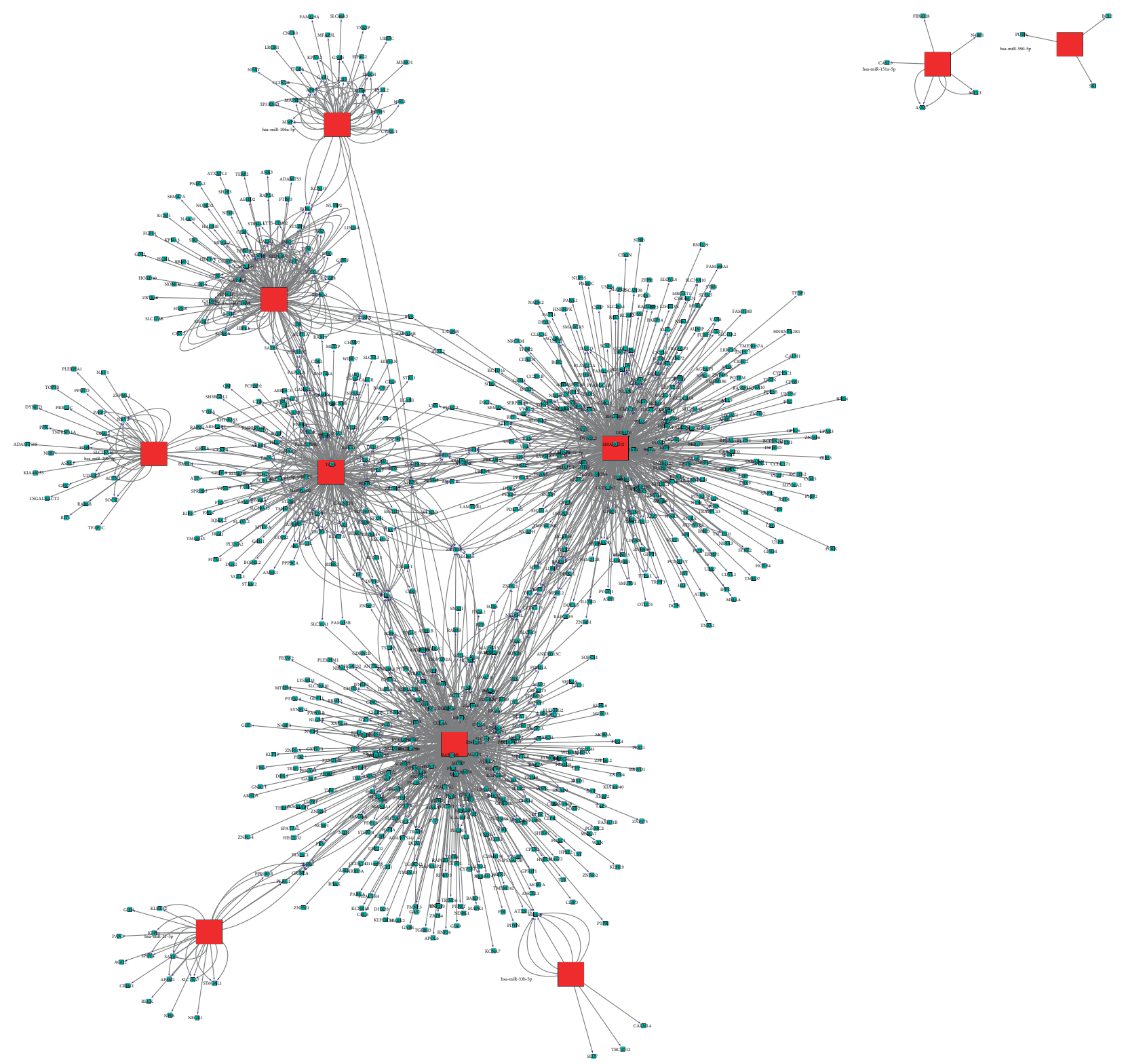

FIGURE 3: miRNA gene interaction network. Red boxes represent miRNAs, and blue square nodes represent the predicted target genes. Edges show the inhibitory effect of a miRNA to its predicted targets. Overexpressed miRNAs hsa-miR-1290 and hsa-miR-33a-5p show the most target mRNAs. hsa-miR-27b-3p and hsa-miR-21-5p have the highest degree of connectivity among underexpressed miRNAs. Degree refers to the contribution of one miRNA to the target genes around it or the contribution of one gene to the miRNAs around it. The key miRNAs and genes in the network always have the highest degrees of connectivity.

hsa-miR-200a-3p regulates EMT-related gene expression, promotes the proliferation of cancer cells by posttranscriptionally regulating cytoplasmic collapsin response mediator, and prevents apoptotic cell death through downregulation of MKK4 [25].

The top downregulated RON overexpression-associated miRNA was hsa-let-7c-3p. hsa-let-7c-3p, a metastasis suppressor, was shown to suppress cell migration and invasion by downregulating K-RAS, MMP11, Bcl-2, CASP3, and PBX3. Moreover, hsa-let-7c-3p directly repressed the cisplatin-activated IL-6/STAT3 prosurvival pathway to modulate chemosensitivity, and transfection of hsa-let-7c restored sensitivity to cisplatin and increased the rate of esophageal squamous carcinoma cellular apoptosis after exposure $[26,27]$.

Increased expression of hsa-miR-31-5p inhibits cell proliferation, migration, and invasion by regulating the Sp1 transcription factor in hepatocellular carcinoma. However, the function of hsa-miR-31-5p was shown to differ, as hsamiR-31-5p was also demonstrated to positively influence cell motility in correlation with metastatic status by regulating PGE2, which was mediated by EP1-ERK-MMP9 signaling. Moreover, comprehensive miRNA expression profiling analysis found that hsa-miR-31-5p is a diagnostic biomarker for pancreatic cancer $[28,29]$. 


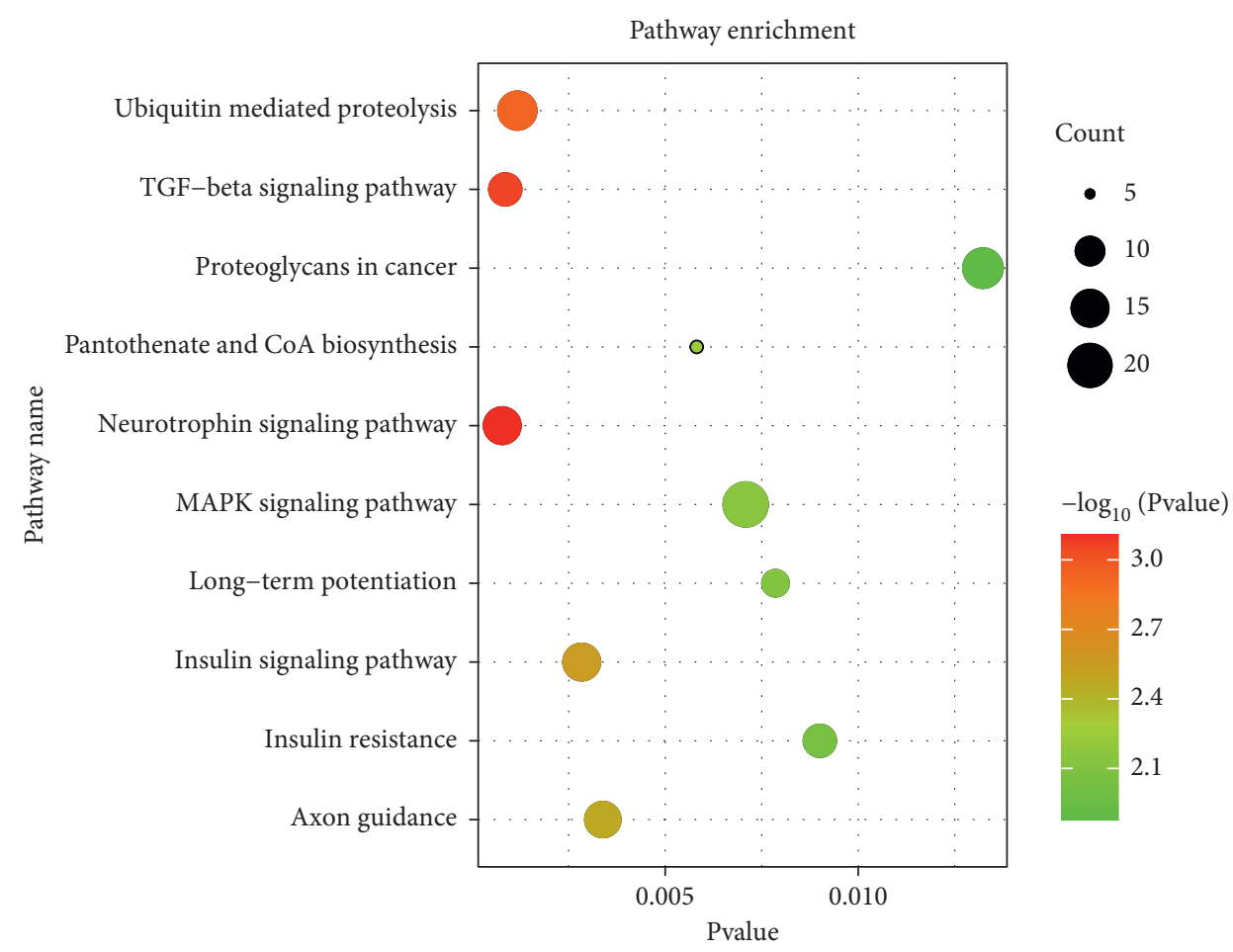

Figure 4: miRNA target pathway enrichment analysis. The top 10 significantly enriched pathways as predicted from target genes of differentially expressed miRNAs in RON overexpressing NSCLC samples.

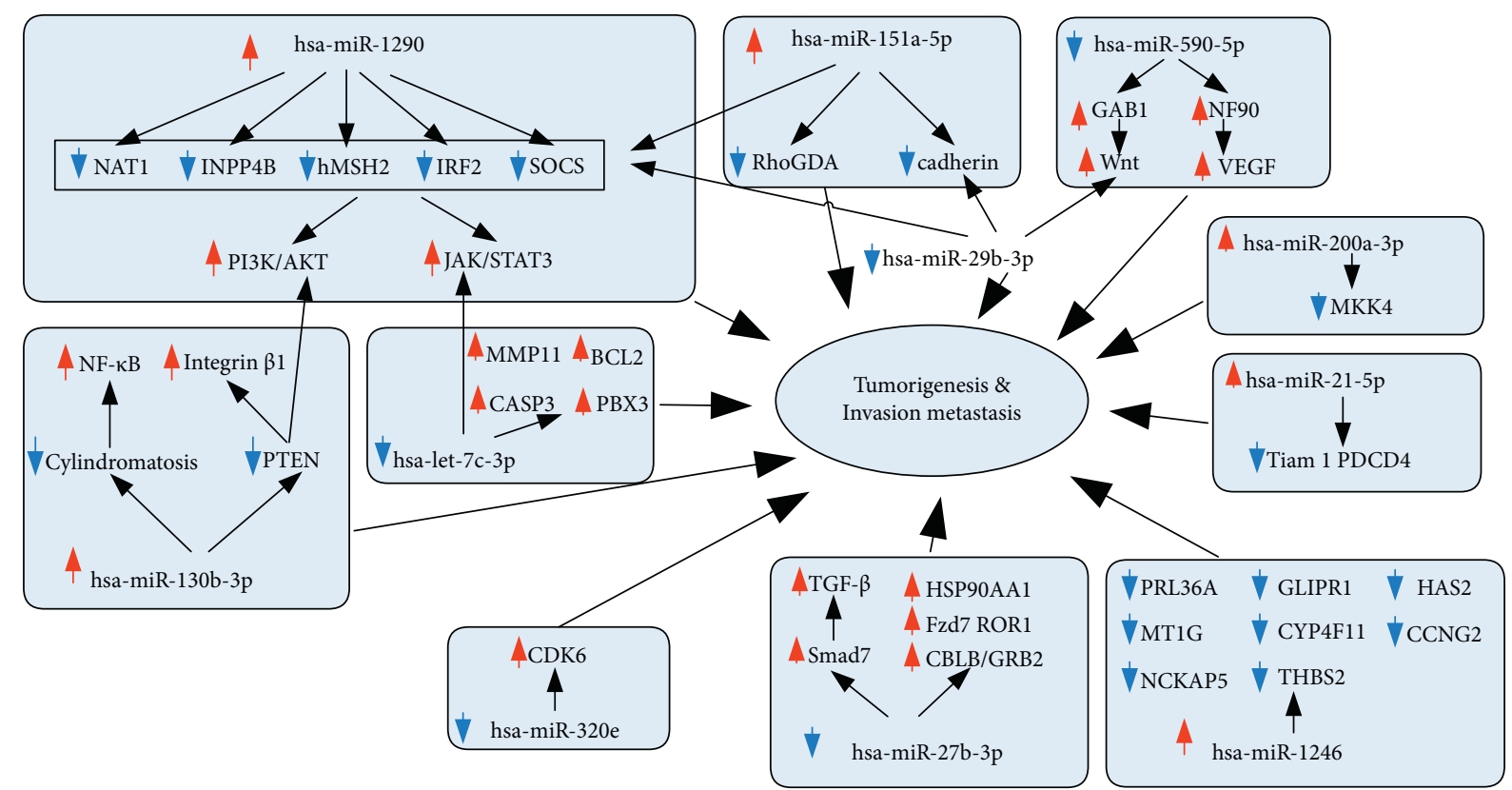

FIGURE 5: A network of RON-related miRNA target genes and potential mechanism of action. Upregulation or downregulation of a specific miRNA or target gene is represented by an upward (red) or a downward (blue) arrow, respectively.

Hsa-let-7a expression plays an important role in tumorigenesis through repressing c-Myc and is significantly downregulated in a number of cancers, including hepatocellular cancer, breast cancer, and ovarian cancer [30].

The hsa-miR-320 family, particularly hsa-miR-320e, is downregulated in colorectal adenoma and affects colorectal tumor proliferation by targeting CDK6. hsa-miR-320e plays an important role in the growth of colorectal tumors and is considered as a biomarker for the early detection of colorectal tumors [31].

Upregulated hsa-miR-27b has a protective role in cell proliferation and migration by targeting Smad7 and 
affecting the TGF- $\beta$ pathway. hsa-miR-27b-3p directly targets HSP90AA1 and Fzd7 in NSCLC, ROR1 in gastric cancer, and CBLB/GRB2 in breast cancer to suppresses cell proliferation, migration, invasion, and expression of MET $[32,33]$.

hsa-miR-29b-3p suppresses cell proliferation, migration, invasion, EMT, and metastasis in vitro and in vivo by regulating DNMT3B, PGRN, and STAT3, elevating E-cadherin expression, and decreasing Snail and vimentin. hsa-miR-29b-3p also represses Wnt signaling, TGF- $\beta 1$ signaling, and STAT3 signaling $[34,35]$.

miR-590-5p inhibits breast cancer cell stemness and metastasis by targeting SOX2, inhibits colorectal cancer angiogenesis and metastasis by regulating the NF90/VEGFA axis, inhibits gastric cancer cell growth and chemosensitivity through the RECK and AKT/ERK pathway, inhibits growth of HepG2 cells via decrease of S100A10 expression and inhibition of Wnt pathway, and suppresses the proliferation and invasion of NSCLC by regulating GAB1 [36, 37].

RON high-expression activates or represses some transcription factors, which likely impacts the expression of a number of miRNAs. The 46 miRNAs that were differentially expressed miRNAs between RON high-expressing and RON nonexpressing NSCLC samples might be important factors that mediate the pathogenesis of NSCLC. These miRNAs promote tumor development via regulation of numerous targets, multiple signaling pathways, and biological functions and behaviors. Among these, EMT and invasion were identified as important functions enriched in these miRNAs, which is consistent with previous findings. Constitutively, high RON expression leads to morphological scattering or stabilized EMT and TGF- $\beta 1$, and activation of the MAPK and Ras pathways was closely related to RONmediated EMT. JAK/STAT, MAPK, and Ras signaling are possible common mediators of the functional behavior of these miRNAs, as the JAK/STAT signaling pathway mediates the biological effects of various external stimuli and controls survival, proliferation, and differentiation of several cell types. RON overexpression may be involved in the pathological process of tumorigenesis through these pathways, although interrogation of the specific mechanisms involved is beyond the scope of this study.

The miRNA expression between NSCLC and SCLC samples was clustered, and a heat map was constructed to visualize miRNA expression patterns. Our findings suggest that while RON expression levels are associated with distinct miRNA expression patterns in NSCLC, the specific lung cancer subtype (either NSCLC or SCLC) has a greater impact on miRNA expression profiles. Furthermore, we analyzed the interactions between miRNAs and their potential target genes that were differentially expressed based on differential RON expression in NSCLC, and we constructed a network of miRNA interactions using Cytoscape software. Our data suggest that the RON-associated miRNAs may impact a number of pathways, including PI3K-Akt, MAPK, endocytosis, proteoglycans in cancer, focal adhesion, and Ras signaling pathway-related activation state; these pathways may be differentially activated between NSCLC samples that exhibit distinct patterns of RON expression and are consistent with previous research which suggested that RON promotes oral squamous cell carcinoma progression by regulating EMT and the MAPK signaling pathway [38].

\section{Conclusion}

In summary, here, miRNA expression patterns of lung cancer associated with differential expression of the human receptor tyrosine kinase RON were profiled. We identified differentially expressed miRNA between NSCLC and SCLC, as well as between NSCLC samples with high RON expression and lacking RON expression. Our results suggest that miRNAs regulating PI3K-Akt, MAPK, endocytosis, proteoglycans in cancer, focal adhesion, and Ras signaling may exhibit significantly different expression patterns associated with differential RON expression in NSCLC. EMT and PI3K-Akt and MAPK signaling pathways are significantly regulated by RON expression levels. The approach and findings of this study may provide new perspectives on the tumor-promoting effect of RON and may lead to the development of improved diagnostic and therapeutic strategies for lung cancer. Further research will verify these differentially expressed miRNAs on larger tissue samples and then evaluate the miRNAs-regulated gene networks predicted by bioinformation analysis. Functional verification of gene-gene interactions can be carried out at both cellular and animal levels.

\section{Data Availability}

The data used to support the findings of this study are available from the corresponding author upon request.

\section{Conflicts of Interest}

The authors declare that they have no conflicts of interest.

\section{Authors' Contributions}

$\mathrm{H} O$ performed the experiment, analyzed data, and wrote the first draft. $\mathrm{K} \mathrm{C}$ reviewed the data and revised the article. $\mathrm{H} \mathrm{W}$ designed the experiment and reviewed the data. All authors read and approved the final version of the article.

\section{Acknowledgments}

This research was supported by a Zhejiang Provincial Natural Science Foundation (LQ20H190001) and opening foundation of the State Key Laboratory for Diagnosis and Treatment of Infectious Diseases, the First Affiliated Hospital, College of Medicine, Zhejiang University (SKLID2020KF042). The authors would like to thank Elixigen (http://med2708.yixie8.com/) for their assistance with English language editing.

\section{References}

[1] W. Pao and N. Girard, "New driver mutations in non-smallcell lung cancer," The Lancet Oncology, vol. 12, no. 2, pp. 175-180, 2011. 
[2] J. Wolf, T. Seto, J.-Y. Han et al., "Capmatinib inMETExon 14mutated orMET-amplified non-small-cell lung cancer," New England Journal of Medicine, vol. 383, no. 10, pp. 944-957, 2020.

[3] D. Dustin, G. Gu, A. R. Beyer et al., "RON signalling promotes therapeutic resistance in ESR1 mutant breast cancer," British Journal of Cancer, vol. 124, no. 1, pp. 191-206, 2021.

[4] J. R. Bourn, S. J. Ruiz-Torres, B. G. Hunt, N. M. Benight, and S. E. Waltz, "Tumor cell intrinsic RON signaling suppresses innate immune responses in breast cancer through inhibition of IRAK4 signaling," Cancer Letters, vol. 503, pp. 75-90, 2021.

[5] T.-H. Weng, M.-Y. Yao, X.-M. Xu et al., "RON and MET Cooverexpression are significant pathological characteristics of poor survival and therapeutic targets of tyrosine kinase inhibitors in triple-negative breast cancer," Cancer Research and Treatment, vol. 52, no. 3, pp. 973-986, 2020.

[6] S. Krishnaswamy, A. K. Mohammed, G. Tripathi, M. S. Alokail, and N. M. Al-Daghri, "Splice variants of the extracellular region of RON receptor tyrosine kinase in lung cancer cell lines identified by PCR and sequencing," BMC Cancer, vol. 17, pp. 738-747, 2017.

[7] C. M. Croce and G. A. Calin, "miRNAs, cancer, and stem cell division," Cell, vol. 122, no. 1, pp. 6-7, 2005.

[8] N. Abd-Aziz, N. I. Kamaruzman, and C. L. Poh, "Development of microRNAs as potential therapeutics against cancer," Journal of oncology, vol. 2020, Article ID 8029721, 14 pages, 2020.

[9] P. T.-W. Yu, A. M. Lowy, and B. Aronow, "RON and c-met differentially regulate microRNA expression during pancreatic carcinogenesis," Journal of the American College of Surgeons, vol. 209, no. 3, p. S118, 2009.

[10] Y.-Q. Chen, Y.-Q. Zhou, J. H. Fisher, and M.-H. Wang, "Targeted expression of the receptor tyrosine kinase RON in distal lung epithelial cells results in multiple tumor formation: oncogenic potential of RON in vivo," Oncogene, vol. 21, no. 41, pp. 6382-6386, 2002.

[11] Y.-Q. Chen, Y.-Q. Zhou, L.-H. Fu, D. Wang, and M.-H. Wang, "Multiple pulmonary adenomas in the lung of transgenic mice overexpressing the RON receptor tyrosine kinase," Carcinogenesis, vol. 23, no. 11, pp. 1811-1819, 2002.

[12] Y.-J. Pan, L.-L. Wei, X.-J. Wu, F.-C. Huo, J. Mou, and D.-S. Pei, "MiR-106a-5p inhibits the cell migration and invasion of renal cell carcinoma through targeting PAK5," Cell Death \& Disease, vol. 8, no. 10, p. e3155, 2017.

[13] S. Dong, X. Zhang, and D. Liu, "Overexpression of long noncoding RNA GAS5 suppresses tumorigenesis and development of gastric cancer by sponging miR-106a-5p through the Akt/mTOR pathway," Biology Open, vol. 8, no. 6, Article ID bio041343, 2019.

[14] W. C. Zhang, T. M. Chin, H. Yang et al., "Tumour-initiating cell-specific miR-1246 and miR-1290 expression converge to promote non-small cell lung cancer progression," Nature Communications, vol. 7, no. 1, p. 11702, 2016.

[15] G. Kim, H.-J. An, M.-J. Lee et al., "Hsa-miR-1246 and hsamiR-1290 are associated with stemness and invasiveness of non-small cell lung cancer," Lung Cancer, vol. 91, pp. 15-22, 2016.

[16] D. Bhagirath, T. L. Yang, N. Bucay et al., "microRNA-1246 is an exosomal biomarker for aggressive prostate cancer," Cancer Research, vol. 78, no. 7, pp. 1833-1844, 2018.

[17] T. Cooks, I. S. Pateras, L. M. Jenkins et al., "Mutant p53 cancers reprogram macrophages to tumor supporting macrophages via exosomal miR-1246," Nature Communications, vol. 9, no. 1, pp. 771-815, 2018.
[18] R. Zhang and T. Xia, "Long non-coding RNA XIST regulates PDCD4 expression by interacting with miR-21-5p and inhibits osteosarcoma cell growth and metastasis," International Journal of Oncology, vol. 51, no. 5, pp. 1460-1470, 2017.

[19] J.-H. He, Y.-G. Li, Z.-P. Han et al., "The CircRNA-ACAP2/ hsa-miR-21-5p/Tiam1 regulatory feedback circuit affects the proliferation, migration, and invasion of colon cancer SW480 cells," Cellular Physiology and Biochemistry, vol. 49, no. 4, pp. 1539-1550, 2018.

[20] K. Sanders, Novel Roles for miR-151a and miR-128 in Cancer, University of California Irvine, Irvine, CA, USA, 2016.

[21] I. Daugaard, K. J. Sanders, A. Idica et al., "miR-151a induces partial EMT by regulating E-cadherin in NSCLC cells," Oncogenesis, vol. 6, no. 7, p. e366, 2017.

[22] X. Cui, C. Kong, Y. Zhu et al., "miR-130b, an onco-miRNA in bladder cancer, is directly regulated by NF- $\kappa \mathrm{B}$ and sustains NF- $\kappa \mathrm{B}$ activation by decreasing Cylindromatosis expression," Oncotarget, vol. 7, no. 30, pp. 48547-48561, 2016.

[23] Y. Shui, X. Yu, R. Duan et al., "miR-130b-3p inhibits cell invasion and migration by targeting the Notch ligand Deltalike 1 in breast carcinoma," Gene, vol. 609, pp. 80-87, 2017.

[24] N. Shakespear, M. Ogura, J. Yamaki, and Y. Homma, "Astrocyte-derived exosomal microRNA miR-200a-3p prevents MPP+-Induced apoptotic cell death through down-regulation of MKK4," Neurochemical Research, vol. 45, pp. 1-14, 2020.

[25] D. Zhou, L. Zhang, W. Sun et al., "Cytidine monophosphate kinase is inhibited by the TGF- $\beta$ signalling pathway through the upregulation of miR-130b-3p in human epithelial ovarian cancer," Cellular Signalling, vol. 35, pp. 197-207, 2017.

[26] L. Wang, J. Li, Y. Li, and L.-B. Pang, "Hsa-let-7c exerts an antitumor function by negatively regulating ANP32E in lung adenocarcinoma," Tissue and Cell, vol. 65, Article ID 101372, 2020.

[27] K. Sugimura, H. Miyata, K. Tanaka et al., "Let-7 expression is a significant determinant of response to chemotherapy through the regulation of IL-6/STAT3 pathway in esophageal squamous cell carcinoma," Clinical Cancer Research, vol. 18, no. 18, pp. 5144-5153, 2012.

[28] J. Mlcochova, P. Faltejskova-Vychytilova, M. Ferracin et al., "microRNA expression profiling identifies miR-31-5p/3p as associated with time to progression in wild-type RAS metastatic colorectal cancer treated with cetuximab," Oncotarget, vol. 6, no. 36, 38704 pages, Article ID 38695, 2015.

[29] G. Zhao, C. Han, Z. Zhang, L. Wang, and J. Xu, "Increased expression of microRNA-31-5p inhibits cell proliferation, migration, and invasion via regulating $\mathrm{Sp} 1$ transcription factor in HepG2 hepatocellular carcinoma cell line," Biochemical and Biophysical Research Communications, vol. 490, no. 2, pp. 371-377, 2017.

[30] Y. Liu, B. Yin, C. Zhang, L. Zhou, and J. Fan, "Hsa-let-7a functions as a tumor suppressor in renal cell carcinoma cell lines by targeting c-myc," Biochemical and Biophysical Research Communications, vol. 417, no. 1, pp. 371-375, 2012.

[31] T. Tadano, Y. Kakuta, S. Hamada et al., "microRNA-320 family is downregulated in colorectal adenoma and affects tumor proliferation by targeting CDK6," World Journal of Gastrointestinal Oncology, vol. 8, no. 7, p. 532, 2016.

[32] J. Tao, X. Zhi, X. Zhang et al., "miR-27b-3p suppresses cell proliferation through targeting receptor tyrosine kinase like orphan receptor 1 in gastric cancer," Journal of Experimental \& Clinical Cancer Research, vol. 34, no. 1, p. 139, 2015.

[33] X. Zeng, C. Huang, L. Senavirathna, P. Wang, and L. Liu, "miR-27b inhibits fibroblast activation via targeting TGF $\beta$ signaling pathway," BMC Cell Biology, vol. 18, no. 1, p. 9, 2017. 
[34] M. Lv, Z. Zhong, M. Huang, Q. Tian, R. Jiang, and J. Chen, "IncRNA H19 regulates epithelial-mesenchymal transition and metastasis of bladder cancer by miR-29b-3p as competing endogenous RNA," Biochimica et Biophysica Acta (BBA) Molecular Cell Research, vol. 1864, no. 10, pp. 1887-1899, 2017.

[35] D. Ding, C. Li, T. Zhao, D. Li, L. Yang, and B. Zhang, "LncRNA H19/miR-29b-3p/PGRN axis promoted epithelialmesenchymal transition of colorectal cancer cells by acting on Wnt signaling," Molecules and Cells, vol. 41, pp. 423-435, 2018 .

[36] Q. Zhou, Y. Zhu, X. Wei et al., "MiR-590-5p inhibits colorectal cancer angiogenesis and metastasis by regulating nuclear factor 90/vascular endothelial growth factor A axis," Cell Death \& Disease, vol. 7, no. 10, p. e2413, 2016.

[37] S. Ekhteraei-Tousi, B. Mohammad-Soltani, M. Sadeghizadeh, S. J. Mowla, S. Parsi, and M. Soleimani, "Inhibitory effect of hsa-miR-590-5p on cardiosphere-derived stem cells differentiation through downregulation of TGFB signaling," Journal of Cellular Biochemistry, vol. 116, no. 1, pp. 179-191, 2015.

[38] S. A. Kim, K. H. Lee, D. H. Lee et al., "Receptor tyrosine kinase, RON, promotes tumor progression by regulating EMT and the MAPK signaling pathway in human oral squamous cell carcinoma," International Journal of Oncology, vol. 55, no. 2, pp. 513-526, 2019. 